\title{
ORIGINAL ARTICLE Cervical multisegmental motor responses in healthy subjects
}

\author{
MA Sabbahi ${ }^{1}$ and YS Sengul ${ }^{2}$
}

Study design: Experimental design.

Objectives: This descriptive design study presents multisegmental motor responses in the upper extremities after stimulation of cervical spinal vertebrae.

Setting: Neuro Lab of Texas Woman's University, School of Physical Therapy, Texas, USA.

Methods: In trial 1, C7 spinal segment was electrically stimulated in 13 healthy subjects using surface electrodes while recording responses from abductor digiti minimi (ADM), abductor pollicis brevis (APB), flexor carpi radialis (FCR) and biceps brachii (BB) in the right upper extremity. In trial 2, C7 stimulation was carried out while recording responses from the APB and BB bilaterally. In trial 3, C7 stimulation was carried out while recording responses from the flexor hallucis brevis (FHB), soleus, vastus medialis (VMO) and gluteus medius (GM) in the right lower extremity. The amplitude of the signal and the deflection latency were the measured parameters. Descriptive statistics were completed on the data.

Results: Results showed response amplitudes in all muscles of the upper extremities ranging from 328 to $1239 \mu \mathrm{V}$, with the largest recorded from the APB and ADM, then the FCR and BB. Muscular responses were recorded simultaneously in bilateral muscles. Response latency was comparable, in bilateral similar muscles, and was varied from 6 to $16.5 \mathrm{msec}$, being longer in the ADM and APB, shorter in FCR and shortest in the BB. No lower limb muscles responded to C7 spinal stimulation, using current setup/method.

Conclusion: These responses appear to be caused by stimulating the dorsal roots or motor nuclei of the cervical region and could be useful in testing patients with cervical spinal disorders.

Spinal Cord (2012) 50, 432-439; doi:10.1038/sc.2011.166; published online 17 January 2012

Keywords: multisegmental responses; spinal reflexes; spinal tracts; spinal stimulation; cervical spinal disorders

\section{INTRODUCTION}

Spinal cord injuries and diseases at the cervical region are 21st century pathologies and the computer age syndrome. The prolonged time sitting with the internet and computer work, longer distance driving habits and increased percent of drink and driving accidents are central factors in increasing cervical spine pathologies. ${ }^{1,2}$ Cervical spondylosis with and without canal stenosis, cervical spinal cord injuries and whiplash injuries are common among young drivers using text messaging. ${ }^{3}$ However, evaluation and treatment of these conditions are based largely on subjective clinical evaluation or imaging studies. Very often, subtle cervical pathologies escape detection because the testing approaches vary; in some, the patient is lying and in others the patient is standing. Imaging studies, besides testing the anatomic variable of the spine it usually test patients in nonfunctional posture (lying position). Furthermore, cervical spinal cord injuries tested by conventional clinical and neurological methods might not be sensitive enough to detect or identify those pathways or fibers that are still available after the injury or those at a subfunctional level. There is a need for more sensitive procedures to identify these pathologies. Electrophysiological testing could fill that need. Recording H-reflexes and F-waves are common procedures, although this approach does not directly test the spinal cord. Because it tests one spinal segment/ muscle at a time, it can be time consuming. The new procedure of recording multisegmental responses, to spinal stimulation, may hold promise for future studies of cervical spine pathology.
A recent study reported that transcutaneous spinal cord stimulation resulted in muscular responses recorded in several lower limb muscles. ${ }^{4,5}$ These responses were called multisegmental reflexes because using a single focal stimulation of the spinal cord, several muscles of different spinal segments were recorded simultaneously. Responses were recorded after T11-12 transcutaneous spinal stimulation. Minassian et al. ${ }^{6}$ described these responses as posterior root-muscle reflexes elicited by transcutaneous stimulation of the human lumbosacral cord. This assumption of Minassian's was supported by the theoretical study of Struijk et al. ${ }^{7}$ To our knowledge, no report has been published about multisegmental responses following cervical spine stimulation.

Cervical spine multisegmental motor responses (CMMR) would be useful in patients with injury of cervical spinal segments as well as other spinal cord injuries and diseases. ${ }^{8}$ The degree of reduction in signal amplitude of a specific muscle would indicate a compromized pathway to such muscle/spinal level that is comparable to the multisegmental motor responses (MMR) amplitude. Multisegmental responses following cervical spine stimulation could also test the physiology and motor control of the cervical spinal cord to the upper and lower limbs. Courtine et al. ${ }^{4}$ evaluated the modulation of these multisegmental responses in leg muscles during walking and running, using T11-12 stimulation. Similarly, cervical multisegmental responses would be useful in testing cervical spinal control of the upper limbs during reaching and other functions. Corticospinal

${ }^{1}$ Texas Woman's University, School of Physical Therapy, Houston, TX, USA and 2Dokuz Eylul University, School of Physical Therapy and Rehabilitation, Izmir, Turkey Correspondence: Professor MA Sabbahi, Texas Woman's University, School of Physical Therapy, 6700 Fannin St., Houston, TX 77030 , USA.

E-mail: msabbahi@twu.edu

Received 12 May 2011; revised 25 August 2011; accepted 25 September 2011; published online 17 January 2012 
pathways as well as other descending pathways pass from the cervical to the thoracolumbar region to control lower limb muscles. It is therefore expected to record lower limb muscular responses by cervical spine stimulation, if these pathways are stimulated by the electrical stimuli. Cervical spinal stimulation and lower limb muscular responses in healthy subjects are, therefore, needed for future comparison with patients with spinal cord disorders/injuries and lower limb dysfunctions.

Epidural spinal cord stimulation was reported to cause stepping-like muscular activities in patients with complete spinal cord injuries. ${ }^{9}$ On the other hand, Gerasimenko ${ }^{10}$ reported that such epidural stimulation of the rat spinal cord elicited early, intermediate and late responses in several extensor and flexor muscles in the hind limb. Further, they showed that those early-evoked responses are, in fact, direct stimulation of the spinal cord and not the dorsal roots. If this is the case with percutaneous cervical spinal stimulation, it could be an effective procedure in management of spinal cord injury.

It is the purpose of this study to report the effect of C7 spinal stimulation using focal electrical stimuli on the muscular responses of the upper and lower limbs in healthy subjects. It is hypothesized that, in healthy subjects, C7 electrical stimulation using surface electrodes will result in muscular responses in several upper limb muscles similar to T11-12 stimulation with lower limb multisegmental motor responses. ${ }^{11}$ It is also hypothesized that muscular responses could be recorded in lower limb muscles with $\mathrm{C} 7$ electrical stimulation in healthy subjects.

\section{MATERIALS AND METHODS}

A total of 13 subjects, 5 men and 8 women aged 20-45 years, signed informed consent approved by the review board of Texas Electrophysiology services, to participate in the present study. All subjects were healthy with no neck or arm pain or radiculopathy during the previous 12 months. Subjects were excluded if they had metabolic or neurological diseases, arthritis or radiculopathy of the cervical spine or cancers. Table 1 presents the demographic characteristics of the study subjects.

\section{Electrical stimulation and recording}

The C7 spinal segment was electrically stimulated using $1 \mathrm{msec}^{2}$ wave pulses at 0.2 pulses per second and at the maximum muscular response. The $\mathrm{C} 7$ segment was located by palpation during flexion/extension of the neck, and a cup electrode was affixed to the intervertebral space between C7 and T1 using $3 \mathrm{M}$ hypoallergenic tape (3M Company, St Paul, MN, USA). For effective stimulation, the electrode (cathode) was kept snug by the operator during testing. The reference electrode (anode) was a 2 -inch square, pre-gelled flexible pad (similar to those used with transcutaneous electrical nerve stimulation), and the pad applied on the left acromion. Stimulation was the most critical step of this experiment. Stimulus intensity was increased until maximum compound action potentials were recorded from the tested muscles at a comfortable level.

Muscular responses/action potentials were recorded using a four-channel Cadwell electromyography unit (Cadwell Lab., Kennewick, WA, USA). Surface silver-silver chloride cup electrodes with electrically conducted gel was applied on the muscles using $3 \mathrm{M}$ hypoallergenic tape. A metal ground electrode with electrically conducted gel was applied to the subject's forearm on the radial head. In trial 1 , action potentials were recorded from the motor points of the following muscles in the right upper extremity: abductor digiti minimi (ADM),

\section{Table 1 Demographic characteristic of patients}

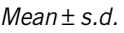

abductor pollicis brevis (APB), flexor carpi radialis (FCR) and biceps brachii (BB). In ADM and APB, active electrode was applied on the ball (motor point) of the muscle with the reference electrode distal to it (on the metacarpophalangeal (MP) joint). In FCR, the active electrode was applied on the motor point, about proximal $1 / 3$ of the line drawn between the medial epicondyle and the radial styloid process, ${ }^{12}$ with the reference electrode $2.5 \mathrm{~cm}$ lateral to it. In $\mathrm{BB}$ muscle, the active and reference electrodes were applied on the ball (motor point) of the muscle, midway between the shoulder and elbow levels. In order to ensure bilateral recording with a single stimuli, trial 2 applied C7 stimulation while recording responses from the $\mathrm{APB}$ and $\mathrm{BB}$ muscles in both upper extremities. Trial 3 included C7 electrical stimulation while recording action potentials from the following muscles of the right lower limbs: flexor hallucis brevis (FHB), soleus, vastus medialis (VMO) and gluteus medius (GM). In FHB, active electrode was applied on the ball (motor point) of the muscle (on the plantar surfaces of first phalanx). In soleus, the active electrode was applied on the motor point, about $4-6 \mathrm{~cm}$ distal to the musculotendinous junction of the gastrocnemius, with the soleus muscle on the posterior midline of the lower limb. The ground electrode was a round metal electrode $(2 \mathrm{~cm}$ diameter applied on the posterior aspect of the calf, proximal to the recording electrode. In VMO, the active electrode was applied on the motor point, about $45^{\circ}$ angles to the longitudinal axis of the femur in line with the orientation of the VMO muscle fibers. The recording bar electrode for the GM was placed on the GM lateral part. Recording parameters were $100-1000 \mu \mathrm{V} \mathrm{div}^{-1}$ with sweep speed of 5 msec div $^{-1}$, using $10 \mathrm{~Hz}-10 \mathrm{kHz}$ bandpass of Butterworth filter.

FCR H-reflexes were also elicited and recorded from the right upper extremity using the method of Sabbahi and Khalil. ${ }^{12}$ In this method, the median nerve was electrically stimulated ( $1 \mathrm{msec}, 0.2$ pulses per second at $\mathrm{H}$-max) at the cubital fossa using a surface electrode with the cathode proximal to the anode. FCR Hreflexes were recorded from the motor point of the muscle at the forearm with the reference electrode placed lateral to the active electrode. A metal ground electrode ( $2 \mathrm{~cm}$ diameter) was applied on the lateral epicondyle. Electrically conducted gel was applied with all stimulation, recording and ground electrodes. Recording parameters were $200-1000 \mu_{\mathrm{div}^{-1}}$ using a filter setting of $10 \mathrm{~Hz}-$ $10 \mathrm{kHz}$. Figure 1 shows the stimulation and recording electrodes' location.

There was some concern that there might be some electric current leakage to the cardiac muscle during cervical electrical stimulation. In order to test that concern, we tested five subjects (three men and two women) using 12 Leads Electrocardiogram (ECG) during progressing electrical stimulation of C7 vertebral level (same location for eliciting cervical MMR). This was recorded during electrical stimulation intensity ranging from $10 \mathrm{~mA}$ to $100 \mathrm{~mA}(10,30$, 50, 80 and 100) (Figure 2). The ECG record was checked by a cardiologist and an ECG technician.

\section{Experimental procedures}

After signing informed consent, subjects were seated in a chair without arms with the forearm resting on a pillow in the lap. Stimulation and recording electrodes sites were cleansed using alcohol and dried; then the electrodes were applied in place. The three trials were conducted in order. Subjects tolerated the electrical stimuli but requested rest periods between trials. Resting times between trials were 3-5 min. Subjects were told to relax during the testing periods and to refrain from head turning or arm movement. FCR H-reflexes were then recorded. At the end of the experiment, electrodes were removed, skin was cleansed and patient was dismissed.

\section{Signal and statistical analyses}

Five traces were recorded and averaged for each muscle at the three trials. Five traces were also recorded for the FCR H-reflexes. The peak-to-peak amplitude and deflection latency were the dependent parameters for the CMMR and H-reflex. Signals were pooled for all subjects using descriptive statistics, with the mean and s.d. analyzed using the SPSS-11.0 (SPSS Inc, Chicago, IL, USA) statistical package. The CMMR and FCR H-reflex latencies were performed using descriptive statistics (mean values and s.d.). Signal was correlated to subject's height and age using Spearman's rho correlation.

\section{RESULTS}

Cervical spinal stimulation showed large and robust responses in all upper limb muscles tested. We recorded these potentials 


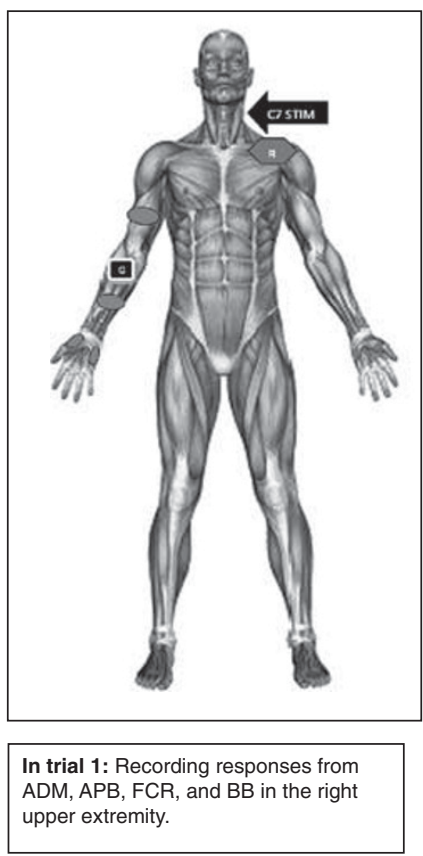

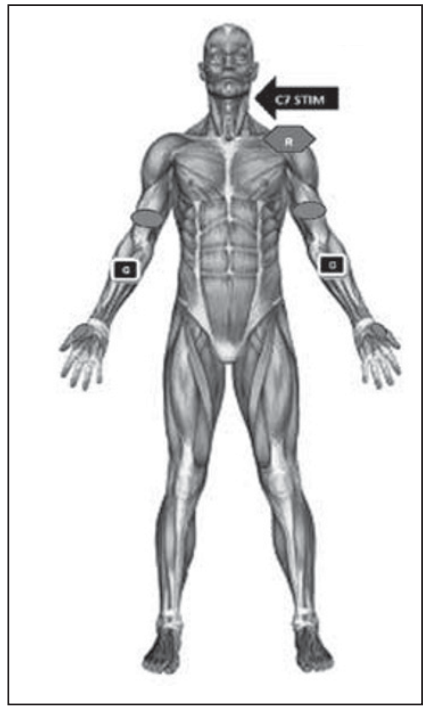

In trial 2: Recording responses from the APB and BB bilaterally

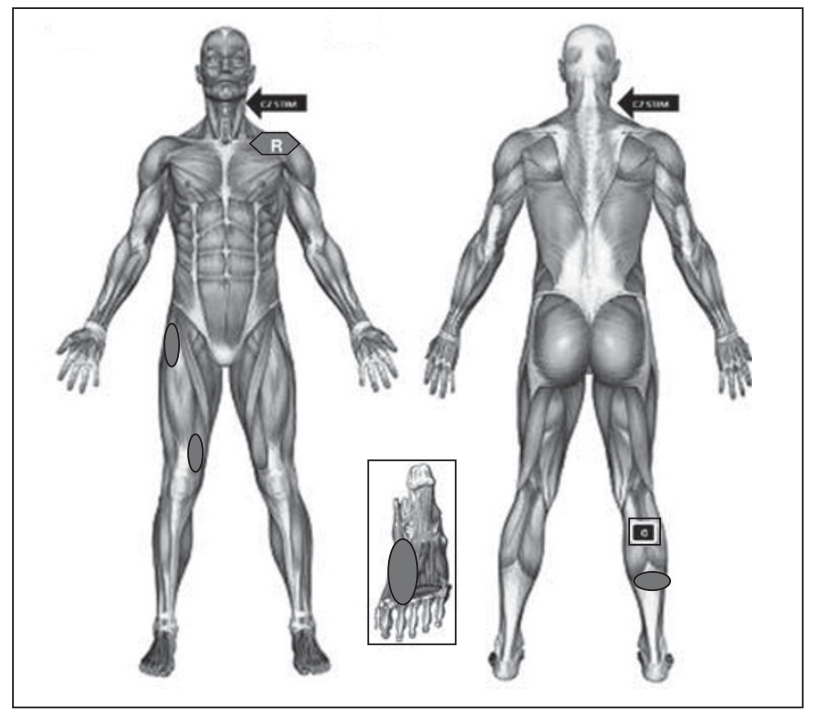

In trial 3: Recording responses from the FHB, soleus, VMO, and GM in the right lower extremity.

Figure 1 Stimulation and recording electrodes location ( $\bigcirc$ : recording electrode,

simultaneously in both upper limbs. However, no responses were recorded in any lower limb muscle with cervical spinal stimulation. Cervical spinal stimulation was slightly less comfortable than T11-12 stimulation reported in our companion study (Thoracolumbar Multisegmental Motor Responses in the Upper and Lower Limbs in Healthy Subjects). ${ }^{11}$ This mild discomfort was only reported by our subjects at higher intensities, although such responses were also recorded at lower stimulus intensities.

\section{C7 stimulation and upper limb responses}

Cervical spinal stimulation resulted in large amplitude muscular responses at the $\mathrm{APB}, \mathrm{ADM}, \mathrm{FCR}$ and $\mathrm{BB}$ muscles with single focal stimuli. Signal amplitude was larger in distal limb muscles, such as APB and $\mathrm{ADM}(1.2 \mathrm{mV}$ in $\mathrm{APB} ; 1.1 \mathrm{mV}$ in $\mathrm{ADM})$ and became smaller for proximal limb muscles, such as FCR and BB $(0.52 \mathrm{mV}$ in FCR; $0.33 \mathrm{mV}$ in BB) (Table 2). The peak-to-peak amplitude of the signal varied greatly between subjects (Table 2, Figure 3). Intra-subjects variability was small (Figure 4). Action potentials were monophasic or biphasic in shape, with duration of 5-18 msec. Action potential duration was always smaller for $\mathrm{BB}$ and FCR than for $\mathrm{ADM}$ and $\mathrm{APB}$ muscles. Response amplitude was generally smaller for the FCR/CMMR than for the FCR H-reflex (Figure 5). Signal amplitude was variable in this study. However, signal reliability and optimization will be the subject of future study.

Latency for action potentials was compatible with the distance between the $\mathrm{C} 7$ stimulation electrode and the muscle (proximal vs distal). Response latencies were $6.08 \mathrm{msec}$ for the $\mathrm{BB}, 8.46 \mathrm{msec}$ for the FCR, $13.6 \mathrm{msec}$ for the ADM and $13.7 \mathrm{msec}$ for the APB (Table 2). Response latency varied on the basis of subject's height. However, response latency variability was substantially smaller than response amplitude (Figures 3 and 6). Response latency for the FCR/CMMR was almost half that of the FCR H-reflex (Figures 4 and 5).

\section{Bilateral recording of multisegmental responses}

Cervical spinal stimulation resulted in simultaneous multisegmental motor responses in both upper limbs. Motor responses from the APB and $\mathrm{BB}$ muscles in both upper limbs can be seen in traces of a sample subject in Figure 7. Table 2 presents the mean and s.d. values for these responses. The amplitude value was slightly higher $(P=0.24)$ in the left, especially for the BB muscles. This can be seen in Figure 3 and Table 2 for all subjects. Inter-subject variability was also high in bilateral recording. Action potential shapes were not the same between the upper limbs muscles.

CMMR response latencies were comparable between the right and left upper limbs and were correlated to the subject's height (Table 3, Figure 6). The degree of correlation was higher for the BB and FCR muscles (proximal) than the APB and ADM muscles (distal).

\section{C7 stimulation and lower limb responses}

Cervical spinal stimulation resulted in no responses in any lower limb muscles (Figure 8) using current electrode setup. A minimal activity might be seen in the VMO at a higher magnification/amplification that might be inconclusive. This lack of response, in most muscles, was recorded for all subjects being tested.

\section{ECG changes with $\mathrm{C} 7$ stimulation}

Results showed no alteration in the ECG pattern in any of the records with all subjects tested (Figure 2). This result indicates safety of cervical spinal stimulation on cardiac function.

\section{DISCUSSION}

C7 spinal stimulation showed large amplitude responses in the ADM, $\mathrm{APB}, \mathrm{FCR}$ and BB muscles. This is probably because of activation of the cervical motor nuclei that innervate the upper limb muscles. A second source for these evoked responses could be dorsal root activation in these upper limb muscles, thus activating homonymous motor nuclei that innervate various upper limb muscles. This is unlikely, though, because of the fact that we used single point stimulation at $\mathrm{C} 7$ that may not directly innervate the $\mathrm{BB}$ with $\mathrm{C} 5$ or the ADM with $\mathrm{C} 8$ segmental levels. ${ }^{13}$ We also applied the stimulation electrode at a central position between the cervical spine protrusions, away from the nerve root exit. This methodological aspect, because of its focal nature, also negates the possibility of electrical stimulation 


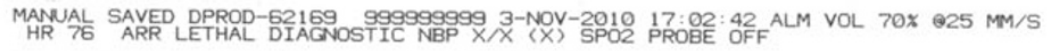
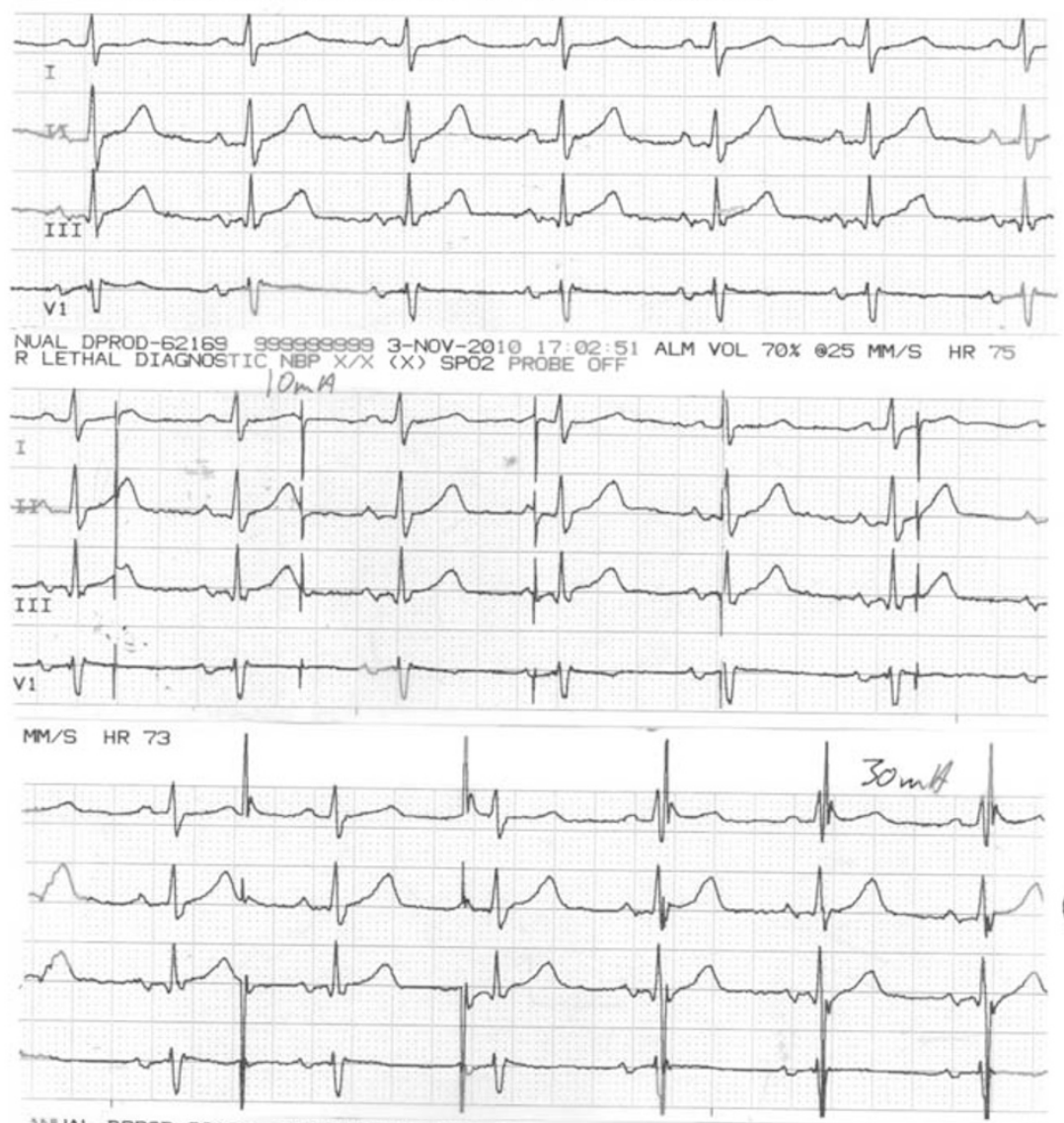

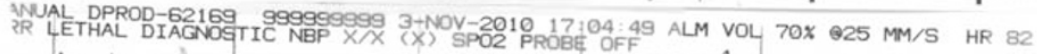
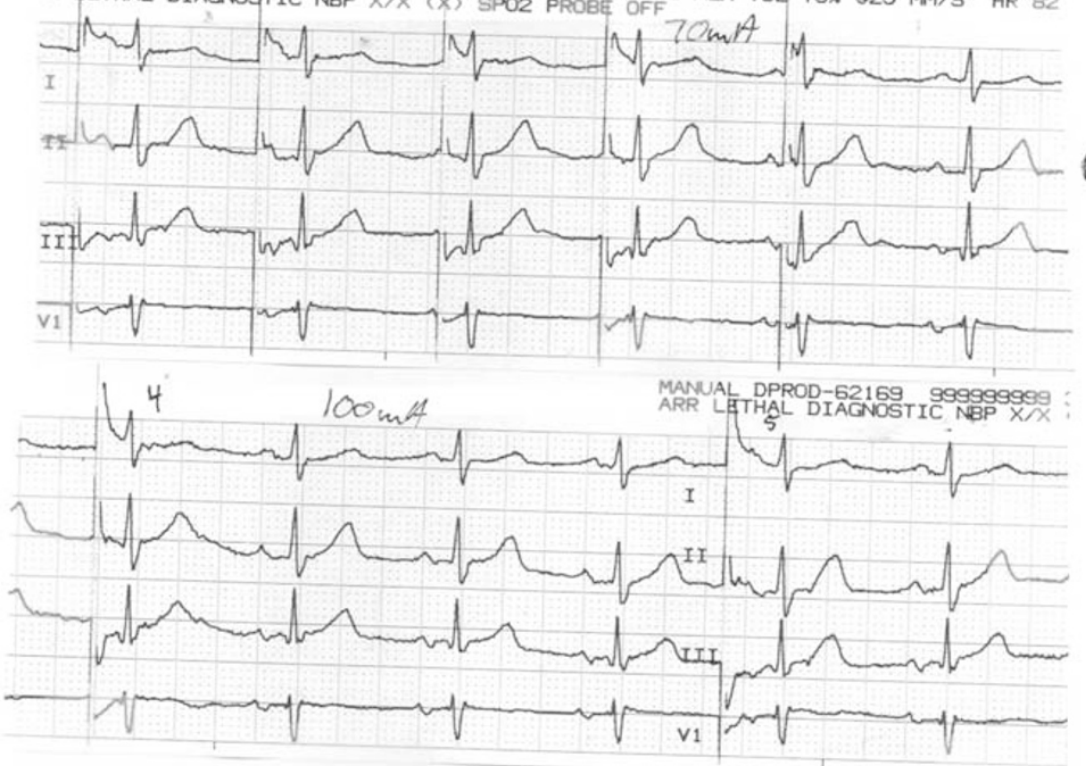

Figure 2 ECG showed no changes with cervical electrical stimulation up to $100 \mathrm{~mA}$. Panel 1 (without stimulation) followed by electrical stimulation at $10 \mathrm{~mA}$ (2), $30 \mathrm{~mA}(3), 70 \mathrm{~mA}$ (4) and $100 \mathrm{~mA}$ (5).

to the motor portion of the nerve root such as those seen in magnetic stimulation. In magnetic stimulation, the operator moves the coil near the nerve root exit and watches for specific muscular response. The magnetic stimulation electrode, because of the size of the coil, appears to be less focal than our stimulation. A detailed analysis of this notion needs to be studied.

The peak-to-peak amplitude of the ADM and APB muscles was the largest compared with proximal limb muscles, such as the FCR and 
Table 2 Amplitude and latency of the CMMR in upper limb muscles following C7 stimulation

\begin{tabular}{|c|c|}
\hline & Mean \pm s.d. \\
\hline \multicolumn{2}{|l|}{$A D M$} \\
\hline Amplitude $(\mu \mathrm{V})$ & $1119.23 \pm 1502.30$ \\
\hline Latency (msec) & $13.62 \pm 1.94$ \\
\hline \multicolumn{2}{|l|}{$A P B$} \\
\hline Amplitude $(\mu \mathrm{V})$ & $1008.46 \pm 1122.05$ \\
\hline Latency (msec) & $13.69 \pm 1.49$ \\
\hline \multicolumn{2}{|l|}{ FCR } \\
\hline Amplitude $(\mu \mathrm{V})$ & $385.39 \pm 370.46$ \\
\hline Latency (msec) & $8.46 \pm 1.05$ \\
\hline \multicolumn{2}{|l|}{$B B$} \\
\hline Amplitude $(\mu \mathrm{V})$ & $328.85 \pm 370.23$ \\
\hline Latency (msec) & $6.08 \pm 1.44$ \\
\hline \multicolumn{2}{|l|}{ FCR-H reflex } \\
\hline Amplitude $(\mu \mathrm{V})$ & $600.0 \pm 565.69$ \\
\hline Latency (msec) & $16.50 \pm 0.71$ \\
\hline \multicolumn{2}{|l|}{$A P B(R)$} \\
\hline Amplitude $(\mu \mathrm{V})$ & $1288.89 \pm 1433.04$ \\
\hline Latency (msec) & $13.33 \pm 0.50$ \\
\hline \multicolumn{2}{|l|}{$A P B(L)$} \\
\hline Amplitude $(\mu \mathrm{V})$ & $1250.0 \pm 825.48$ \\
\hline Latency (msec) & $13.11 \pm 0.60$ \\
\hline \multicolumn{2}{|l|}{$B B(R)$} \\
\hline Amplitude $(\mu \mathrm{V})$ & $761.11 \pm 834.33$ \\
\hline Latency (msec) & $6.44 \pm 0.53$ \\
\hline \multicolumn{2}{|l|}{$B B(L)$} \\
\hline Amplitude $(\mu \mathrm{V})$ & $1211.11 \pm 946.96$ \\
\hline Latency (msec) & $6.33 \pm 0.71$ \\
\hline
\end{tabular}

Abbreviations: ADM, abductor digiti minimi; $A P B$, abductor pollicis; $A P B(L)$, left abductor pollicis; $A P B(R)$, right abductor pollicis; $B B$, biceps brachii; $B B(L)$, left biceps brachii; $B B(R)$, right biceps brachii; CMMR, cervical spine multisegmental motor responses; FCR, flexor carpi radialis.

BB. This is probably because of increased effectiveness of stimulation intensity at $\mathrm{C} 7$ and $\mathrm{C} 8$ spinal nuclei that are closer to the electrode than BB motor nuclei (C5). Another possibility is the difference in the structural and functional characteristics of the hand muscles (APB and ADM). Hand muscles may contain more motor units with increasing motor activation in dexterity functions, compared with forearm or arm muscles. ${ }^{14} \mathrm{C} 7$ stimulation appears to cause localized activation at the C7 spinal cord segment (causing activation of the FCR and APB muscles), with excitation traveling to the upper C5 and lower C8 spinal segments, resulting in motor discharge to the $\mathrm{BB}$ and $\mathrm{ADM}$ muscles. This is besides the fact that each tested muscle is supplied with more than single spinal level, and the spreading of the stimulation to adjacent segment might result in the recorded response. Alstermark et al. ${ }^{15}$ reported that C3-4 interneurons relay voluntary commands to forelimb muscles in cats and this spinal segment extends caudally for several segments via a propriospinal pathway to the lower cervical, and even the first thoracic spinal segment. In cats, lesions at this spinal segment that does not interrupt the corticospinal pathway

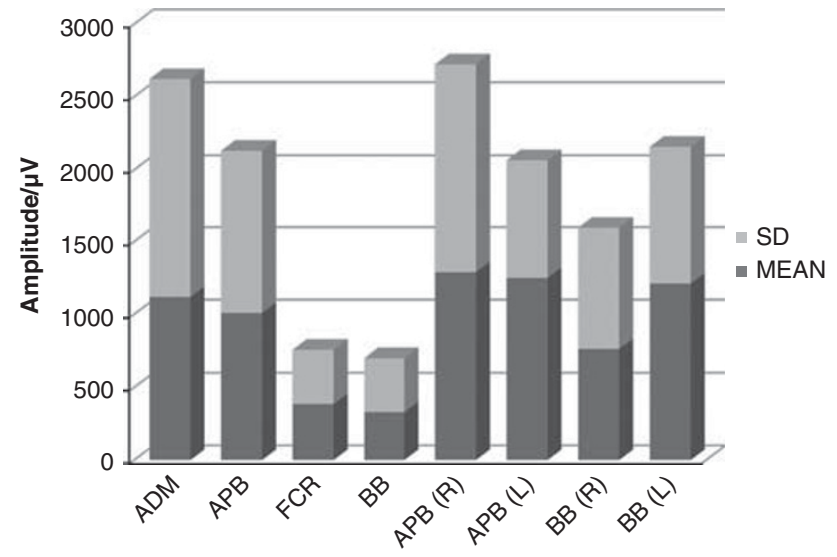

Figure 3 Amplitude and variability of CMMR in upper limb muscles following C7 stimulation. ADM, abductor digiti minimi; APB, abductor pollicis; FCR, flexor carpi radialis; $B B$, biceps brachii; $A P B(R)$, right abductor pollicis; $A P B(L)$, left abductor pollicis; $B B(R)$, right biceps brachii; $\mathrm{BB}(\mathrm{L})$, left biceps brachii.

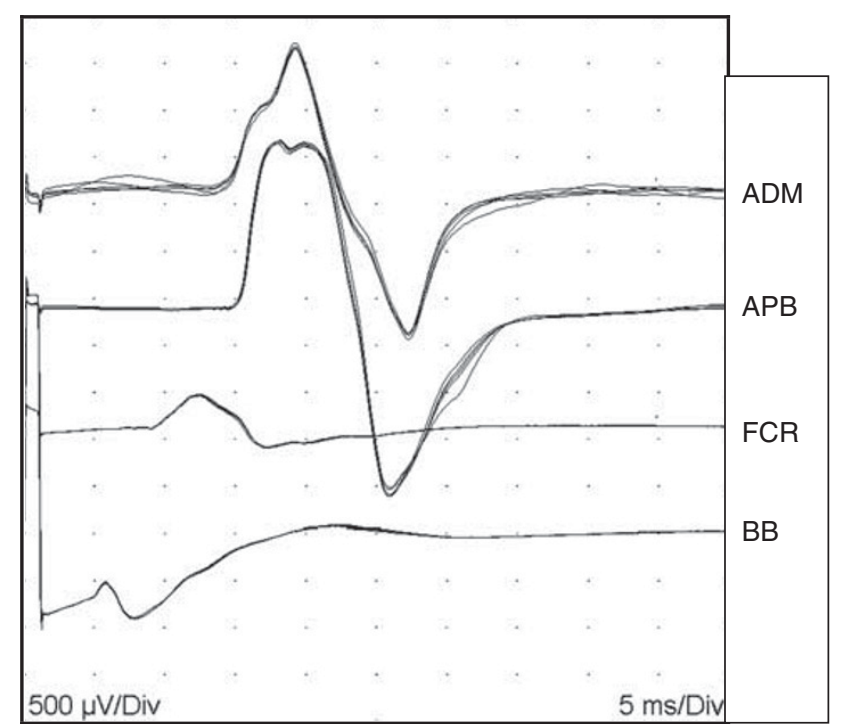

Figure 4 Sample CMMR tracings of upper limb muscles following C7 stimulation. ADM, abductor digiti minimi; $A P B$, abductor pollicis; FCR, flexor carpi radialis; BB, biceps brachii.

does not cause paralysis of limb muscles but it compromises the aiming function of the cat's forelimb. ${ }^{16}$ The authors hypothesized that this wrist movement command is mediated by cortical and rubrospinal systems acting in parallel with C3-4 interneuron link. A similar propriospinal interneuron arrangement that controls wrist movement in humans was proposed by Malmgren and Pierrot-Deseilligny ${ }^{17}$ and could be the underlying pathway for our recorded CMMR in the upper limbs.

Focal C7 spinal stimulation resulted in bilateral motor responses in the APB and BB muscles (right and left limb side). These results confirm the assumption that these responses are due to activation of the dorsal roots supplying bilateral motor nuclei in the cervical cord, although activation of motor nuclei cannot be ruled out. Dorsal root stimulation may result in the activation of the muscles supplied by the stimulated nerve roots with no activation of the contralateral limb. 


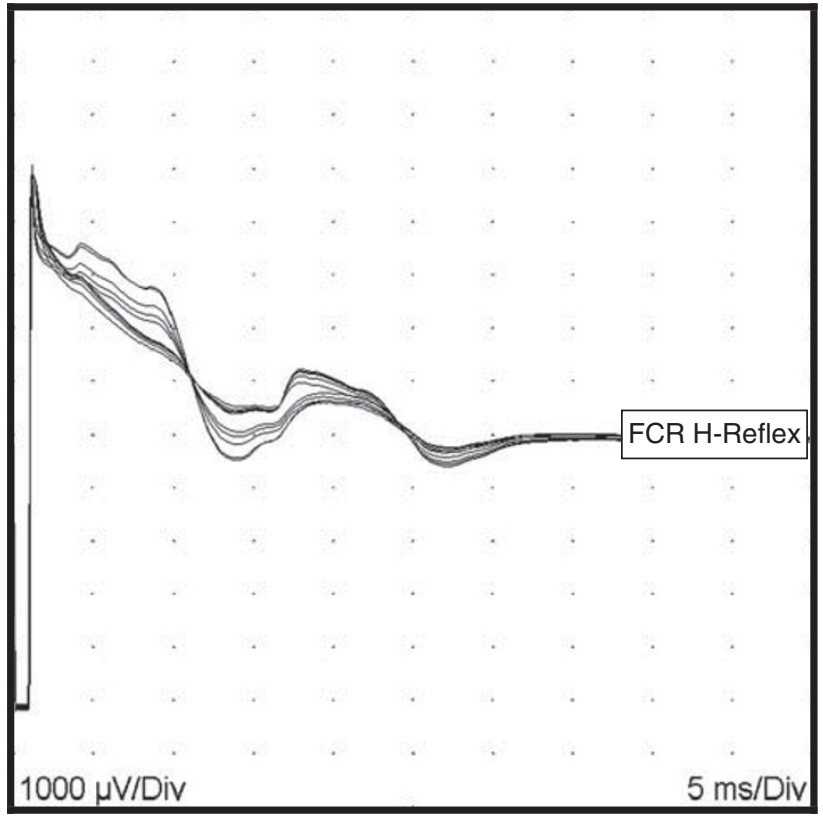

Figure 5 FCR H-reflex.

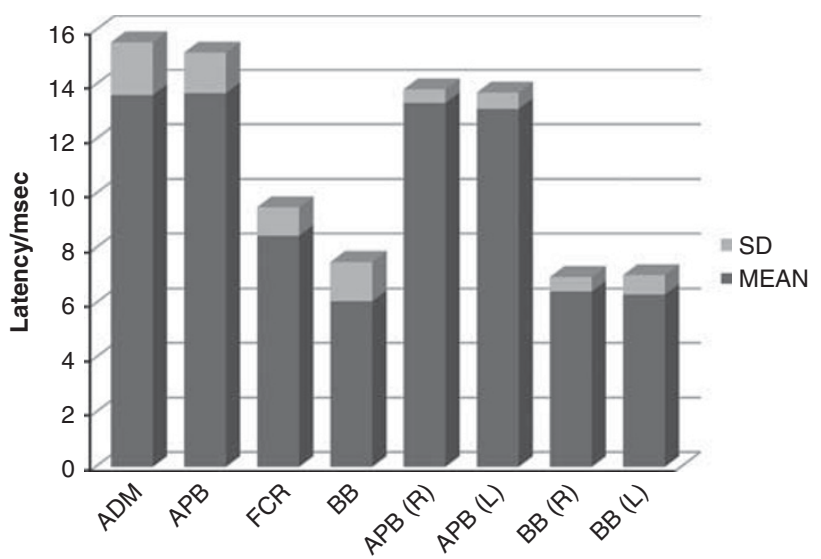

Figure 6 Latency and variability of CMMR in upper limb muscles following C7 stimulation. ADM, abductor digiti minimi; APB, abductor pollicis; FCR, flexor carpi radialis; $B B$, biceps brachii, $A P B(R)$, right abductor pollicis; $A P B(L)$, left abductor pollicis; $B B(R)$, right biceps brachii; $B B(L)$, left biceps brachii.

Response amplitude was slightly larger on the left side than the right side in the APB than BB, probably because of laterality and hand/arm dominance. Another possibility is the field effect caused by applying the reference electrode on to the left acromion near the BB muscle on the left side. It is probably not due to the higher intensity stimuli delivered to the left side motor nuclei over the right side. We were careful to position the stimulation electrode at the central line, in between the upper and lower spinal processes. Hand dominance was not considered, but may be the focus of future studies.

Response latencies were compatible with the proximal-distal location of the muscles tested, being longer for the APB and ADM (hand muscles), shorter for the FCR and shortest for the BB muscles. This latency reflects the travel time from the spinal cord segment to the activated muscle. The latency value indicates that those are direct responses, especially when compared with the latency of the

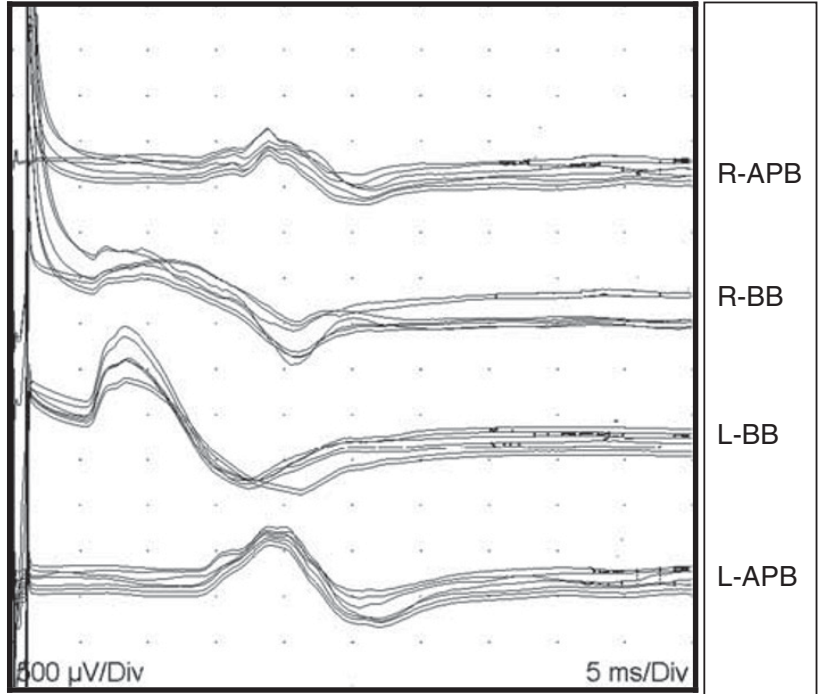

Figure 7 CMMR tracings simultaneously recorded from muscles in both upper limbs with a single C7 stimulation. R-APB, right abductor pollicis brevis; R-BB, right biceps brachii; L-APB, left abductor pollicis brevis; L-BB, left biceps brachii).

Table 3 Correlation between height, weight and latency in upper limb muscles with C7 stimulation

\begin{tabular}{|c|c|c|c|c|c|c|}
\hline \multirow[t]{2}{*}{ Latency } & \multicolumn{2}{|c|}{ Age } & \multicolumn{2}{|c|}{ Height } & \multicolumn{2}{|c|}{ Weight } \\
\hline & $\rho$ & $\mathrm{P}$ & $\rho$ & $\mathrm{P}$ & $\rho$ & $P$ \\
\hline ADM & -0.20 & 0.52 & 0.55 & 0.05 & 0.23 & 0.46 \\
\hline APB & -0.31 & 0.30 & 0.34 & 0.25 & 0.14 & 0.66 \\
\hline FCR & -0.01 & 0.97 & 0.11 & 0.72 & 0.17 & 0.58 \\
\hline BB & -0.04 & 0.89 & -0.08 & 0.80 & 0.14 & 0.63 \\
\hline
\end{tabular}

ADM, abductor digiti minimi; APB, abductor pollicis; BB, biceps brachii; FCR, flexor carpi radialis; $\rho$, Spearman's correlation coefficient.

$P$ indicates $P$-value of the Spearman rank correlation.

FCR H-reflex. The CMMR/FCR response of $8.5 \mathrm{msec}$ was half that of the FCR H-reflex (Figure 5), supporting this assumption. No Hreflexes could be recorded from other muscles in upper limbs of relaxed subjects. ${ }^{12}$ Response latency was comparable with bilateral recording for the $\mathrm{APB}$ and $\mathrm{BB}$, and lend more support to direct spinal cord stimulation than to dorsal root stimulation. Root stimulation may result in longer latency on one side than the other.

Response latency for the APB was comparable with that of the ADM muscle. With comparable length of the median and ulnar nerves (and their medial and lateral cords) from the spinal cord to the hand, an almost equal latency indicated direct activation of the spinal nuclei and equal conduction velocity (Table 2). We did not measure the distance between the cervical spinal segment and the hand to determine the conduction velocity value. This will be the focus of future studies. However, the response latency was moderately correlated to the subject's height.

The action potential duration for the CMMR of the APB and ADM was longer than those of the BB and FCR muscles. This was probably because of increased dispersion of motor unit activities in the hand versus the forearm and arm muscles. Such dispersion would be more important to dexterity function than torque function generated by the 


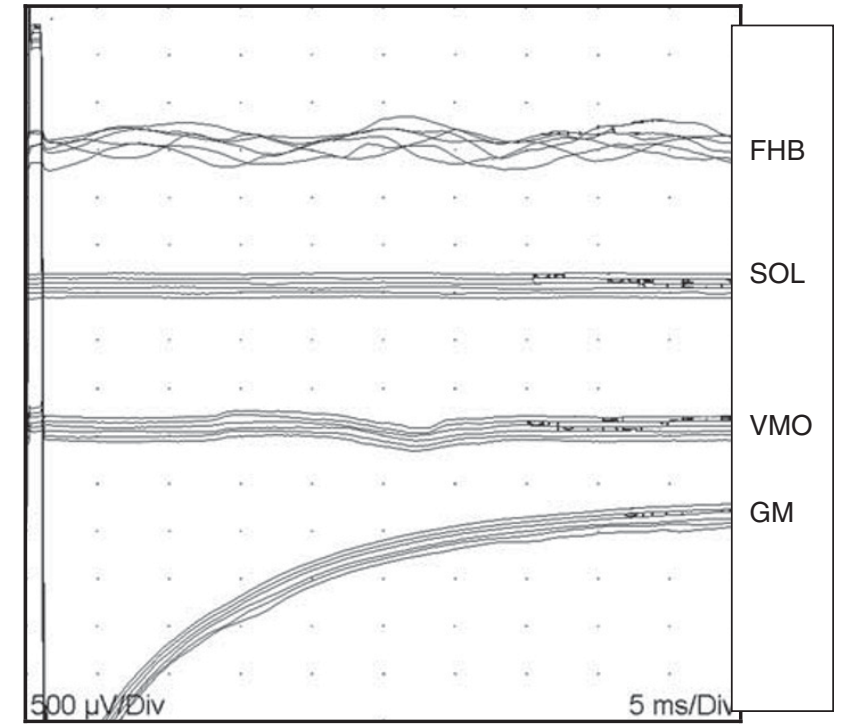

Figure 8 No CMMR was recorded in any of the lower limb muscles following C7 stimulation. FHB, flexor hallucis brevis; SOL, soleus; VMO, vastus medialis obloquies; GM, gluteus medius.

proximal limb muscles. Another possibility for dispersion in the action potential duration is the fact that $\mathrm{ADM}$ and $\mathrm{APB}$ were the most distal limb muscles that we tested. The signal had to travel farther through varying nerve trunks and peripheral nerves with varying conduction velocities. The longer duration potential represents the velocity variation with the time taken to reach the target muscle fiber/motor unit.

CMMR in any of the lower limb muscles, using current electrodes setup. This indicates that the stimulation did not activate the corticospinal pathways or any other descending pathways that formed a synapse to the lumbar motor nuclei. ${ }^{18,19}$ In our companion study, ${ }^{11}$ we used lower stimulation intensity to electrically stimulate the T11-12 spinal segment, and large amplitude motor responses were recorded in upper limb muscles, indicating possible connections between thoracolumbar segments and the motor nuclei of the upper limb muscles, but it appears that such cervical segmental connections with lower limb muscles ${ }^{20}$ is limited using current method of testing. If these responses are transmitted through propriospinal pathways, as proposed in our companion study, it means that there is no descending propriospinal pathway, or possible limited pathway, between the cervical and lumbar spinal segments. ${ }^{21}$ In fact, this has been reported by Kiernan ${ }^{22}$ and lends support to our assumption.

This study used percutaneous stimulation to the spinal cord segments. This cervical spinal stimulation was comfortable to our subjects, especially at lower stimulation intensities, with no recorded side effects. It is considered more convenient, safe and could be easily applied in the clinic when compared with the more invasive intraspinal stimulation. ${ }^{23}$ The variability in the amplitude of the CMMR need further studies to identify its source (methodological or physiological). Recent studies showed lower variability of the MMR responses with methodological modifications. This will be the focus of future publication.

The clinical validity of these cervical MMR needs to be compared with those of carried out with magnetic stimulation. Although magnetic stimulation is not painful but muscular responses are specific to a single muscle for each trial, and to our knowledge no published studies showed multisegmental responses to magnetic stimulation. Furthermore, magnetic stimulation technique is more costly and electrical stimulation such as those seen in the MMR can be incorporated in conventional electromyography studies using available equipment. Cervical MMR results in multisegmental muscular responses mostly in the upper limbs. In this report, we were able to record muscular responses from four muscles at each trial. However, hypothetically these responses could be recorded from all muscles of the upper limb simultaneously. This provides a comparison of functional/electrical integrity of several spinal segments simultaneously. A pathology at one spinal level could be clinically detected in the reduced amplitude of its muscular response when compared with the muscular responses of other muscles for example, APB as compared with ADM, FCR, BB and triceps. An on-going pilot study showed the sensitivity of these MMR procedures to indentify the pathological level of spinal cord injury and extent of such injury. This will be the focus of future report.

The origin of these MMR potentials remains to be identified. Patient's studies might help in such investigations.

These muscular responses could be useful in testing patients with spinal cord injury at the cervical level, identifying (C5-C8 levels). It may also be useful in testing patients with spinal disorders of motoneuronal origin, such as motoneurone diseases or post-polio syndrome and patients with degenerative cervical radiculopathy. This will be the focus of future studies.

These results are limited by the small number of subjects and muscles tested, as well as the inability to record muscular activities in the lower limb at an acceptable level of amplification. Furthermore, a better method for stability and effectiveness of the electrical stimulation is necessary and will be the focus for future studies.

\section{CONCLUSIONS}

Focused C7 spinal stimulation, using current method and electrodes setup, resulted in multisegmental motor responses in upper limb muscles but not in lower limb muscles. This may be due to motor nuclei stimulation that activated upper limb muscles to direct motoneuronal-axonal-muscular responses. These responses could be useful procedures for testing patients with cervical spinal disorders of motoneuronal origin.

\section{DATA ARCHIVING}

There was no data to deposit.

\section{CONFLICT OF INTEREST}

The authors declare no conflict of interest.

\section{ACKNOWLEDGEMENTS}

Dr Mohamed Jerrodi and Dr Josh Sulik helped with the ECG tests and interpretation of the records.

1 National Spinal Cord Injury Statistical Center. Spinal cord injury: facts and figures at a Glance. February 2010 (online).

2 Cote P, van der Velde G, Cassidy JD, Carroll LJ, Hogg-Johnson S, Holm LW et al. The burden and determinants of neck pain in workers: results of the Bone and Joint Decade 2000-2010 Task Force on Neck Pain and Its Associated Disorders. Spine (Phila Pa 1976) 2008; 33(4 Suppl): S60-S74.

3 McCartt AT, Hellinga LA, Bratiman KA. Cell phones and driving: review of research. Traffic Inj Prev 2006; 7: 89-106.

4 Courtine G, Harkema SJ, Dy CJ, Gerasimenko YP, Dyhre-Poulsen P. Modulation of multisegmental monosynaptic responses in a variety of leg muscles during walking and running in humans. J Physiol 2007; 582(Pt 3): 1125-1139. 
5 Kitano K, Koceja DM. Spinal reflex in human lower leg muscles evoked by transcutaneous spinal cord stimulation. J Neurosci Methods 2009; 180: 111-115.

6 Minassian K, Persy I, Rattay F, Dimitrijevic MR, Hofer C, Kern H. Posterior root-muscle reflexes elicited by transcutaneous stimulation of the human lumbosacral cord. Muscle Nerve 2007; 35: 327-336.

7 Struijk JJ, Holsheimer J, Boom HB. Excitation of dorsal root fibers in spinal cord stimulation: a theoretical study. IEEE Trans Biomed Eng 1993; 40: 632-639.

8 Edgerton VR, Tillakaratne NJ, Bigbee AJ, de Leon RD, Roy RR. Plasticity of the spinal neural circuitry after injury. Annu Rev Neurosci 2004: 27: 145-167.

9 Minassian K, Jilge B, Rattay F, Pinter MM, Binder H, Gerstenbrand F et al. Steppinglike movements in humans with complete spinal cord injury induced by epidural stimulation of the lumbar cord: electromyographic study of compound muscle action potentials. Spinal Cord 2004; 42: 401-416.

10 Gerasimenko YP, Lavrov IA, Courtine G, Ichiyama RM, Dy CJ, Zhong H et al. Spinal cord reflexes induced by epidural spinal cord stimulation in normal awake rats. $J$ Neurosci Methods 2006; 157: 253-263.

11 Sabbahi MA, Sengul YS. Thoracolumbar multisegmental motor responses in the upper and lower limbs in healthy subjects. Spinal Cord 2011; 49: 741-748.

12 Sabbahi MA, Khalil M. Segmental H-reflex studies in upper and lower limbs of healthy subjects. Arch Phys Med Rehabil 1990; 71: 216-222.

13 Moore KDA. Clinically Oriented Anatomy, 5th edn Lippincott Williams \& Wilkins: New York, 2006.

14 Johnson MA, Polgar J, Weightman D, Appleton D. Data on the distribution of fibre types in thirty-six human muscles. An autopsy study. J Neurol Sci 1973; 18: 111-129.
15 Alstermark B, Lundberg A, Pettersson LG. The pathway from la forelimb afferents to the motor cortex: a new hypothesis. Neurosci Res 1991; 11: 221-225.

16 Alstermark B, Lundberg A, Norrsell U, Sybirska E. Integration in descending motor pathways controlling the forelimb in the cat. 9. Differential behavioural defects after spinal cord lesions interrupting defined pathways from higher centres to motoneurones. Exp Brain Res 1981; 42: 299-318.

17 Malmgren K, Pierrot-Deseilligny E. Evidence for non-monosynaptic la excitation of human wrist flexor motoneurones, possibly via propriospinal neurones. J Physiol 1988; 405: 747-764.

18 Ghez C. The control of movement. In: Kandel ER SJ, Jessell TM (eds). Principles of Neural Science, 3rd edn Elsevier Science: New York, 1991 pp 540-541.

19 Jankowska E, Lundberg A, Roberts WJ, Stuart D. A long propriospinal system with direct effect on motoneurones and on interneurones in the cat lumbosacral cord. Exp Brain Res 1974; 21: 169-194.

20 Matsushita M, Ikeda M, Hosoya Y. The location of spinal neurons with long descending axons (long descending propriospinal tract neurons) in the cat: a study with the horseradish peroxidase technique. J Comp Neurol 1979; 184: 63-80.

21 Molenaar I, Kuypers HG. Cells of origin of propriospinal fibers and of fibers ascending to supraspinal levels. A HRP study in cat and rhesus monkey. Brain Res 1978; 152: 429-450.

22 Kiernan J. Barr's The Human Nervous system- An anatomical Viewpoint. 8th edn Lippincott Williams \& Wilkins New York, 2005.

23 Gaunt RA, Prochazka A, Mushahwar VK, Guevremont L, Ellaway PH. Intraspinal microstimulation excites multisegmental sensory afferents at lower stimulus levels than local alpha-motoneuron responses. J Neurophysiol 2006; 96: 2995-3005.

\section{Editorial Note on: Neurophysiological assessment of spine disorders: old fashion techniques for modern clinical problems}

Spinal Cord (2012) 50, 439; doi:10.1038/sc.2011.173; published online 17 January 2012

Editorial Note on: Spinal Cord (2012) 50, 432-439; doi:10.1038/ sc.2011.166; published online 17 January 2012

Magnetic Resonance Imaging (MRI) was developed in the 1980's and has revolutionized the clinical evaluation of patients with spine disorders: nowadays most spine disorders are easily diagnosed by expert neuroradiologists. However, a complete anatomo-functional characterization of the spine - an thus a correct diagnosis - can be obtained only including clinical neurophysiological examinations (electromyography and evoked potentials among others) and correlating MRI and neurophysiological findings with the patient's signs and symptoms. Hithererto, not all patients with spine disorders receive a prompt and correct diagnosis. Moreover, in some cases neurophysiological examinations are important not only for the diagnosis, but also for the monitoring and follow-up.

Sabbahi and Sengul ${ }^{1}$ propose, in this issue, that multisegmental responses following cervical spine electrical stimulation could be a useful tool for testing patients with cervical spinal disorders. Highintensity electrical stimulation delivered over the spine is painful. In principle, this technique could be used to provide information about nerve roots ${ }^{2,3}$ (and about cauda equina with electric shocks delivered over the lumbar spine at even higher intensity). ${ }^{4}$ However, the same information about nerve roots can be obtained in a nonpainful way with magnetic stimulation. ${ }^{3,5}$ The drawback of this nonpainful assessment is the higher price of magnetic stimulators compared with the electrical ones. Its by-product is that magnetic stimulators can also be used to stimulate the motor cortex in order to quickly and safely study the central motor conduction time, which is widely used to assess spine disorders. ${ }^{5}$

Certainly there is a need for optimizing the neuroradiological and neurophysiological assessment of spine disorders, but painful techniques have much less possibility to enter the armamentarium used in daily clinical practice. Sabbahi and Sengul thus have-in the next future - the hard task to demonstrate that multisegmental response is really a useful tool for 'testing patients with cervical spinal disorders'.

\section{CONFLICT OF INTEREST}

The author declares no conflict of interest.

A Oliviero

FENNSI Group, Hospital Nacional de Parapléjicos, SESCAM, Toledo, Spain

E-mail: antonioo@sescam.jccm.es

1 Sabbahi MA, Sengul YS. Cervical multisegmental motor responses in healthy subjects. Spinal Cord 2012; 50: 432-439.

2 Mills KR, Murray NM. Electrical stimulation over the human vertebral column: which neural elements are excited? Electroencephalogr Clin Neurophysiol 1986; 63: 582-589.

3 Ugawa Y, Rothwell JC, Day BL, Thompson PD, Marsden CD. Magnetic stimulation over the spinal enlargements. J Neurol Neurosurg Psychiatry 1989; 52: 1025-1032.

4 Maertens de Noordhout A, Rothwell JC, Thompson PD, Day BL, Marsden CD. Percutaneous electrical stimulation of lumbosacral roots in man. $J$ Neurol Neurosurg Psychiatry 1988; 51: 174-181.

5 Di Lazzaro V, Oliviero A, Profice P, Ferrara L, Saturno E, Pilato F et al. The diagnostic value of motor evoked potentials. Clin Neurophysiol 1999; 110: 1297-1307. 